\title{
A Low-Field Mobility Model for Bulk and Ultrathin-Body SOI p-MOSFETs With Different Surface and Channel Orientations
}

\author{
Luca Silvestri, Susanna Reggiani, Member, IEEE, Elena Gnani, Member, IEEE, \\ Antonio Gnudi, Member, IEEE, and Giorgio Baccarani, Fellow, IEEE
}

\begin{abstract}
An easy-to-implement hole mobility model, which accurately predicts low-field mobility in bulk MOSFETs and ultrathin-body (UTB) silicon-on-insulator FETs with different crystal orientations, is developed. The model accounts for the influence of the surface orientation and the inplane current-flow direction on effective masses, subband repopulation, and scattering rates. The effects induced by extremely small silicon thicknesses are also addressed. A good agreement with the experimental mobilities of bulk and UTB FETs with silicon thicknesses from $60 \mathrm{~nm}$ to values as small as about 2.7 and $2.3 \mathrm{~nm}$ is demonstrated for devices with (100) and (110) substrates, respectively.
\end{abstract}

Index Terms-Crystal orientation, mobility model, siliconon-insulator (SOI) MOSFETs, ultrathin silicon.

\section{INTRODUCTION}

$\mathbf{T}$ HE AGGRESSIVE downscaling of CMOS devices is reaching intrinsic limitations and needs new technological solutions. Ultrathin-body (UTB) devices such as fin-shaped FETs, trigate FETs, and silicon nanowires are the most promising candidates for fabricating sub-50-nm devices [1]. Among their features, they usually exhibit a sidewall transport on the (110) crystallographic planes [2], [3]. Thus, a deep comprehension of the physical details related with the different crystallographic orientations is required. The study of the device performance requires predictive physical models for the carrier transport. To this purpose, both low-field mobility models [4] and enhanced drift-diffusion models, which account for the quasi-ballistic transport, have been proposed [5]. It has been experimentally demonstrated that the low-field electron and hole mobilities are sensitive functions of the silicon-body thickness, particularly when $t_{\mathrm{Si}}$ is below $5 \mathrm{~nm}$ [6], [7]. On one hand,

Manuscript received May 12, 2010; revised September 1, 2010; accepted September 10, 2010. Date of publication October 18, 2010; date of current version November 19, 2010. This work was supported by the European Network of Excellence under Grant 216171 (NANOSIL) via the Italian University Nanoelectronics Team Consortium. The review of this paper was arranged by Editor J. C. S. Woo.

L. Silvestri is with the Ercole De Castro Advanced Research Center on Electronic Systems, University of Bologna, 40136 Bologna, Italy (e-mail: 1silvestri@arces.unibo.it).

S. Reggiani, E. Gnani, A. Gnudi, and G. Baccarani are with Ercole De Castro Advanced Research Center on Electronic Systems and the Department of Electronics, Computer Science and Systems (DEIS), University of Bologna, 40136 Bologna, Italy (e-mail: sreggiani@arces.unibo.it; egnani@arces.unibo.it; agnudi@arces.unibo.it; gbaccarani@arces.unibo.it).

Color versions of one or more of the figures in this paper are available online at http://ieeexplore.ieee.org.

Digital Object Identifier 10.1109/TED.2010.2078821 the electron mobility in UTB MOSFETs has been extensively investigated, and a technology computer-aided design (TCAD) model has been recently proposed in [8] and [9]. On the other hand, to the authors' knowledge, a number of experimental investigations can be found on UTB single-gate silicon-oninsulator (SG-SOI) p-MOSFETs, along with theoretical microscopic analyses accounting for full-band structures and the most relevant scattering mechanisms [10]-[12], but a physically based analytical model for the hole low-field mobility is still missing.

The aim of this paper is to derive a TCAD mobility model suitable for device simulation tools, which accurately predicts the low-field hole mobility in bulk and UTB FETs with different surface and channel orientations, and silicon thicknesses from bulk-like to values as small as $2.3 \mathrm{~nm}$. As the low-field mobility concept implies a uniform device subject to a vanishing electric field in the current-flow direction, the investigation carried out in this paper is focused on long-channel FETs. The analysis of the role played by the effective mobility in short-channel FETs is beyond the scope of this paper [13].

In the following, a complete description of the model is provided, and its validation against experiments is illustrated. The mobility formulation is given in Section II. The modeling of the scattering contributions, namely, the acoustic- and optical-phonon, coulomb, surface-roughness, and interfacestate scattering, is described in Section III. An accurate analytical description of the energy subbands is reported in Section IV. The additional effects needed for ultrathin SOI are shown in Section V. A review of the complete model is reported in Section VI, and the conclusions are finally drawn in Section VII.

\section{Generalized Mobility Model}

Consider a silicon film on a substrate with one of the three crystallographic orientations indicated in Fig. 1 (bottom). The $z$-axis is set parallel to the structural confinement direction, whereas the carrier transport occurs along the $x$-axis. The inversion-layer quantization, due to the combined effects of the structural confinement and the application of a transverse electric field, causes the formation of energy subbands split in three different groups: 1) heavy hole (HH); 2) light hole (LH); and 3) split-off subbands. The latter ones have not been considered in this paper because of their lower energy, which makes them practically unpopulated [10]. The complex shape 

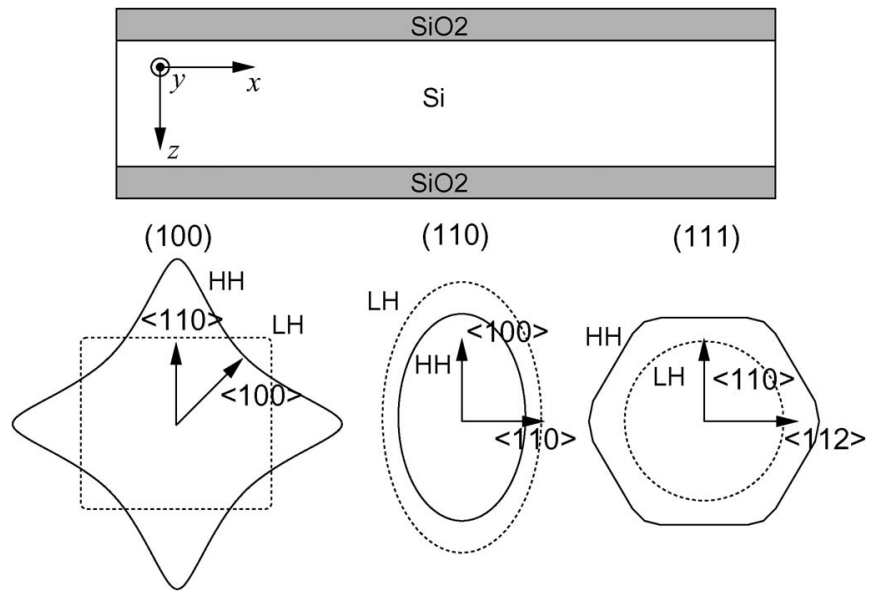

Fig. 1. (Top) Orientation of the reference axes. (Bottom) Inplane minima projections for (100), (110), and (111) surface orientations. The most relevant inplane current flow directions are also indicated.

of the valence-band valleys makes the analytical calculation of the principal effective masses quite problematic. Therefore, a simplified approach has been followed. The masses $m_{z}$ along the quantization direction for the (100)-, (110)-, and (111)-oriented wafers have been extracted by comparing the valley edges calculated by means of a six-band $\mathbf{k} \cdot \mathbf{p}$ approach reported in [10] with the well-known analytical expression determined for a triangular well by Stern and Howard [14], which reads

$$
E_{V_{v}}=\left(\sqrt[3]{\frac{9}{32}}+\sqrt[3]{\frac{9}{4}}\right) \sqrt[3]{\frac{\hbar^{2} q^{2}}{m_{z_{v}}}} E_{\mathrm{eff}}^{2 / 3}
$$

where $\hbar$ is the reduced Planck constant, $q$ is the elementary charge, $E_{\text {eff }}$ is the transverse effective field, and $m_{z_{v}}$ is the quantization mass relative to the $v$ th valley. In the following, $v=1$ and $v=2$ indicate the $\mathrm{LH}$ and $\mathrm{HH}$ valleys, respectively. The comparison of (1) with the $\mathbf{k} \cdot \mathbf{p}$ results is illustrated in Fig. 2, while the extracted quantization masses are reported in Table I. In order to calculate the 2-D density-of-state effective masses, different approaches have been used for the three considered crystallographic orientations. More specifically, in the (100) and (111) orientations, circular parabolic inplane bands for the $\mathrm{HH}$ and LH valleys have been assumed. Within such approximation, the $\mathrm{HH}$ and $\mathrm{LH}$ density-of-state effective masses have been extracted by comparing the analytical calculations of the relative-valley populations as functions of the effective field with the numerical data reported in [10]. For the analytical calculations, the Boltzmann statistics has been assumed, i.e.,

$$
p_{v}=\frac{m_{d_{v}} \exp \left(-E_{V_{v}} / k_{B} T\right)}{\sum_{v^{\prime}=1}^{2} m_{d_{v^{\prime}}} \exp \left(-E_{V_{v^{\prime}}} / k_{B} T\right)}
$$

where $k_{B}$ is the Boltzmann constant and $T$ the lattice temperature. The comparison of the analytical $p_{v}$ with numerical results is reported in Fig. 3.

Different from the cases described above, in the (110) case, a clear inplane anisotropic energy distribution can be observed [see Fig. 1 (bottom)]. In this case, elliptical parabolic inplane bands for the $\mathrm{HH}$ and $\mathrm{LH}$ valleys have been used, and the ef-

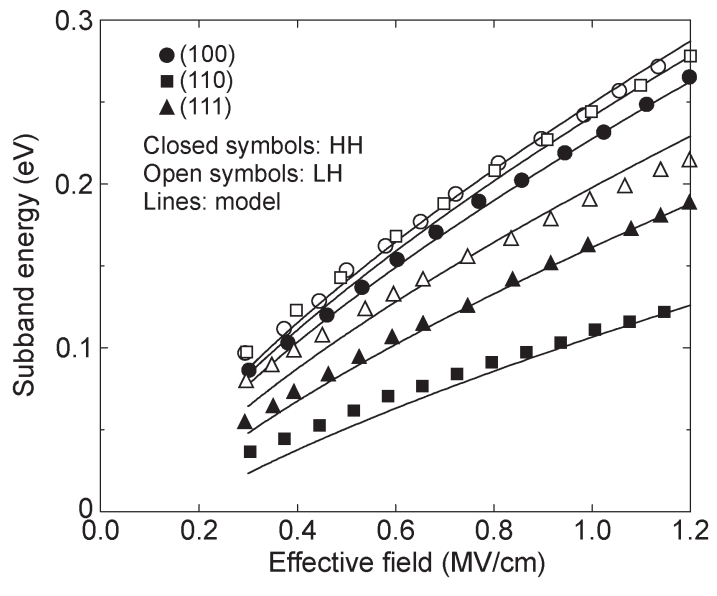

Fig. 2. Energy of the valley edges as a function of the effective field for (100), (110), and (111) wafers. (Symbols) Numerical computations from [10]. (Solid lines) Equation (1) with a shift of $-44 \mathrm{meV}$.

fective masses along the transport $m_{x}$ and the device width $m_{y}$ directions have been extracted by a comparison with the band calculations reported in [15], as shown in Fig. 4. The extracted effective masses are reported in Table I. Finally, the densityof-state effective masses in the (110) case have been calculated as $m_{d_{v}}=\sqrt{m_{x_{v}} m_{y_{v}}}$, and their values have been validated by comparing the analytical $p_{v}$ with the corresponding numerical results shown in [10] (see Fig. 3).

The inplane transport is generally described by a 2-D tensorial effective mobility, which retains the anisotropy of the singlevalley effective mobilities, i.e.,

$$
\hat{\mu}_{\mathrm{eff}}=\sum_{v=1}^{2} p_{v} \hat{\mu}_{v}
$$

A similar formulation is already available in 3-D drift-diffusion transport simulation tools, which handle bulk piezoresistivity and, in general, material anisotropy (see, e.g., [16]). Such tools can be also directly used in this case, given that the out-ofplane (normal to the interface) mobility component plays no role and provided that the inplane effective mobility model (3) is implemented. Unfortunately, this approach is unpopular in commercial tools because the effective mobility dependence on integral (nonlocal) carrier concentration and electric field may lead to numerical problems. As an alternative, a local mobility tensor $\hat{\mu}$, which depends on the local normal electric field $E_{\perp}(z)$ and the hole concentration $n(z)$, can be defined, satisfying the following equation:

$$
\hat{\mu}_{\mathrm{eff}}=\frac{\int_{0}^{t_{\mathrm{Si}}}\left(n(z)-n_{0}(z)\right) \hat{\mu}\left(n, E_{\perp}(z)\right) d z}{\int_{0}^{t_{\mathrm{Si}}}\left(n(z)-n_{0}(z)\right) d z}
$$

where $\left(n(z)-n_{0}(z)\right)$ is the excess carrier concentration in the inversion layer. It should be noted that the experiments measure only the $x x$ component of the mobility tensor. We follow a two-step procedure in the model development. In the first step, we define an analytical model for the effective mobility (3) as a function of $E_{\text {eff }}$ and the inversion-charge concentration per 
TABLE I

EFFECTIVE MASSES FOR A 2-DHG IN (100)-, (110)-, AND (111)-ORIENTED SAMPLES

\begin{tabular}{|c|c|c|c|c|c|}
\hline (Wafer)/<channel $>$ & $m_{z}$ & $m_{x}$ & $m_{y}$ & $m_{d}$ & valleys \\
\hline$(100)$ & $0.25 m_{0}$ & $0.245 m_{0}$ & $0.245 m_{0}$ & $0.245 m_{0}$ & 1 (LH) \\
& $0.29 m_{0}$ & $0.43 m_{0}$ & $0.43 m_{0}$ & $0.43 m_{0}$ & 2 (HH) \\
\hline$(110) /\langle 110\rangle$ & $0.23 m_{0}$ & $0.274 m_{0}$ & $0.348 m_{0}$ & $\sqrt{m_{x} m_{y}}=0.309 m_{0}$ & $1(\mathrm{LH})$ \\
& $1.8 m_{0}$ & $0.122 m_{0}$ & $0.215 m_{0}$ & $\sqrt{m_{x} m_{y}}=0.162 m_{0}$ & $2(\mathrm{HH})$ \\
\hline$(110) /\langle 100\rangle$ & $0.23 m_{0}$ & $0.348 m_{0}$ & $0.274 m_{0}$ & $\sqrt{m_{x} m_{y}}=0.309 m_{0}$ & $1(\mathrm{LH})$ \\
& $1.8 m_{0}$ & $0.215 m_{0}$ & $0.122 m_{0}$ & $\sqrt{m_{x} m_{y}}=0.162 m_{0}$ & $2(\mathrm{HH})$ \\
\hline$(111)$ & $0.41 m_{0}$ & $0.244 m_{0}$ & $0.244 m_{0}$ & $0.244 m_{0}$ & $1(\mathrm{LH})$ \\
& $0.67 m_{0}$ & $0.454 m_{0}$ & $0.454 m_{0}$ & $0.454 m_{0}$ & $2(\mathrm{HH})$ \\
\hline
\end{tabular}

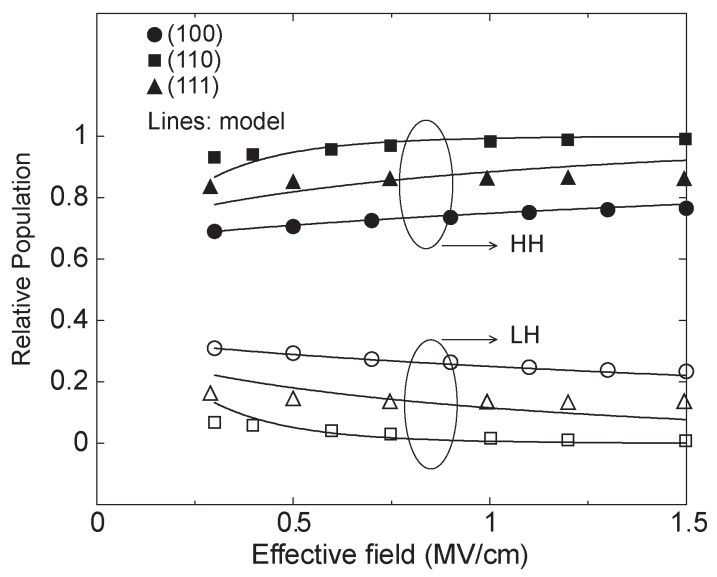

Fig. 3. Relative-valley populations as a function of the effective field for (100), (110), and (111) wafers. (Symbols) Numerical calculations by [10]. (Solid lines) Equation (2).

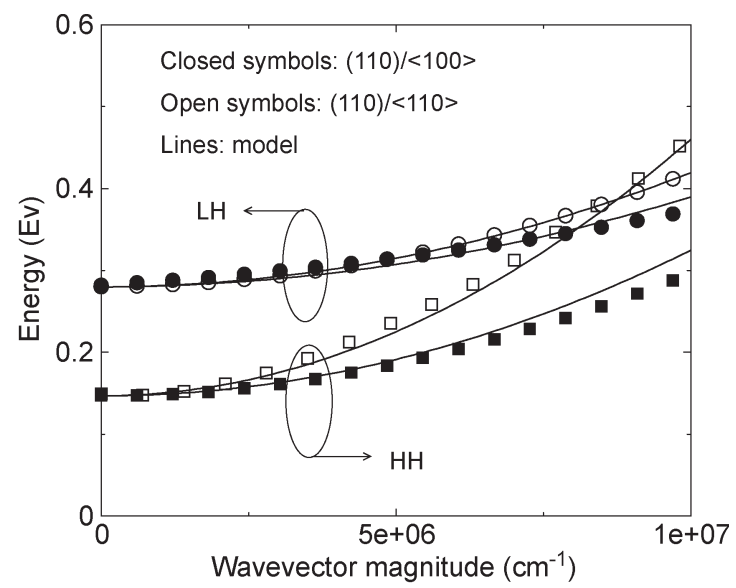

Fig. 4. Energy dispersion relations in (110) wafers obtained using (symbols) the $\mathbf{k} \cdot \mathbf{p}$ approach [15] and (lines) the parabolic band approximation. $\langle 110\rangle$ and $\langle 100\rangle$ inplane crystal directions have been used to separately extract $m_{x}$ and $m_{y}$.

unit area, calibrating the parameters on the experiments. $E_{\text {eff }}$ is calculated as

$$
E_{\mathrm{eff}}=\frac{\int_{0}^{t_{\mathrm{Si}}}\left(n(z)-n_{0}(z)\right) E_{\perp}(z) d z}{\int_{0}^{t_{\mathrm{Si}}}\left(n(z)-n_{0}(z)\right) d z}
$$

In the second step, a local mobility model, which depends on $E_{\perp}(z)$ and $n(z)$, is provided, as described in Section VI. As far as the relative-valley populations are concerned, (2) is used. In (2), the subband edges $E_{V_{v}}$ can be calculated as given by (1) when a bulk MOSFET structure is considered. A different approach needs to be followed when a thin silicon film is addressed, as will be shown in Section IV. Following [17], the single-valley mobility tensor is modeled as

$$
\hat{\mu}_{v}=\mu_{v} \hat{m}_{v}^{-1}, \quad \hat{m}_{v}^{-1}=\left(\begin{array}{cc}
m_{0} / m_{x_{v}} & 0 \\
0 & m_{0} / m_{y_{v}}
\end{array}\right)
$$

where $m_{0}$ is the free-electron mass and $\hat{m}_{v}^{-1}$ is the inverse normalized mass tensor of a 2-D hole gas (2-DHG), defined for each valley $v$ to account for the anisotropy effects induced by different inplane crystal directions. Note that, when (100) or (111) samples are considered, $m_{x_{v}}=m_{y_{v}}=m_{d_{v}}$. Finally, $\mu_{v}$ is calculated by accounting for the different scattering mechanisms combined via Matthiessen's rule, i.e.,

$$
\mu_{v}=\frac{q}{m_{0} \sum_{j} \tau_{v_{j}}^{-1}} .
$$

In (7), $\tau_{v_{j}}$ represents the average momentum relaxation time (MRT) due to the $j$ th scattering mechanism for the $v$ th valley. The average MRTs are illustrated in the next sections.

\section{SCATtERING CONTRibutions}

Following the approach in [8], the acoustic-phonon-limited inverse MRT relative to the $v$ th valley is calculated as

$$
\frac{1}{\tau_{A C, v}}=\frac{C_{v} m_{d_{v}}}{W_{v}}
$$

where $W_{v}$ is the effective width of the hole distribution in the $v$ th valley and $C_{v}$ is a constant related to the intravalley acoustic-phonon scattering parameters. Intervalley scattering is not considered in view of the low-field regime (vanishing longitudinal electric field), which is close to the equilibrium. The average effective widths $W_{v}$ are modeled as in [8], and their calibration has been carried out against the numerical predictions of the Schrödinger-Poisson solver [18], generalized to SG-SOI FETs with different crystal orientations. The eigenfunctions are zero at the $\mathrm{Si} / \mathrm{SiO}_{2}$ interfaces. Neumann boundary conditions for the potential are applied to the bottom edge of the buried oxide in SG-SOI FETs.

The (110) phonon-limited mobility both from numerical calculations [10] and experiments [7] showed a trend in $E_{\text {eff }}$, which differs significantly from the usual $E_{\text {eff }}^{-1 / 3}$ (see Fig. 5). At low temperatures, such a trend is indeed no longer observed. This may suggest that optical phonons can limit mobility at low $E_{\text {eff }}$ values and high temperatures when the two lowest $\mathrm{HH}$ bands and the first $\mathrm{LH}$ band are close enough to allow 


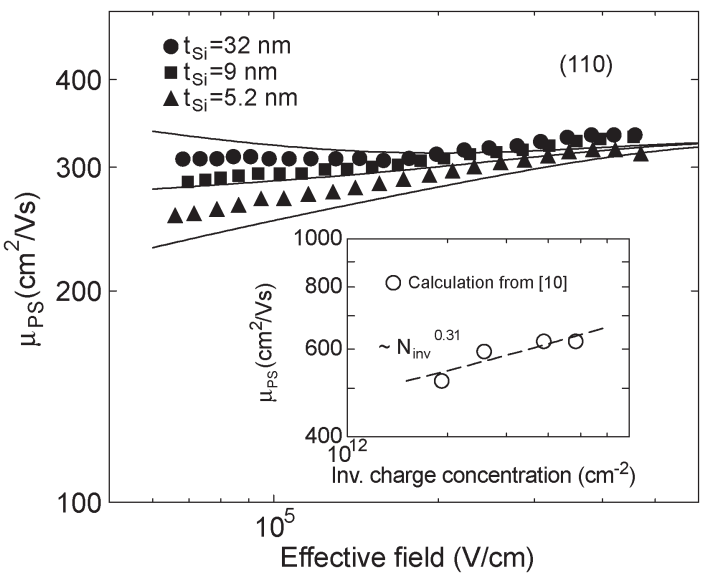

Fig. 5. Phonon-limited hole mobility versus effective field for the $(110) /\langle 110\rangle$ surface extracted (symbols) from experiments in [7] and (lines) from our model. (Inset) $\mu_{\mathrm{PS}}$ versus $N_{\text {inv }}$ from simulations in [10].

intersubband transitions assisted by the absorption of optical phonons to play a role. On the contrary, when the confinement induced by $E_{\text {eff }}$ becomes stronger, this process is weakened by the distance of the energy levels, and the phonon-limited mobility increases until a usual decreasing trend with $E_{\text {eff }}$ is recovered again [10]. In order to model this effect, the opticalphonon-limited inverse MRT relative to the HH band in (110) wafers has been accounted for as

$$
\frac{1}{\tau_{\mathrm{OP}, 2}}=C_{\mathrm{OP}} m_{d_{2}}\left(\frac{E_{\text {effo }}}{E_{\text {eff }}}\right)^{\zeta}
$$

where $\zeta=0.31$ has been extracted from calculations in [10] [see Fig. 5 (inset)] and $C_{\mathrm{OP}}$ is a fitting parameter. The phononlimited mobility relative to the $\mathrm{HH}$ valley has been calculated by combining the scattering term in (9) with (8) via (7). In Fig. 5, the calculated total phonon-limited mobility curves are compared with those extracted from experiments in [7] with different $t_{\mathrm{Si}}$ values. The details on the modeling of the optical-phonon scattering as a function of $t_{\mathrm{Si}}$ are discussed in Section V.

The coulomb and surface-roughness scattering terms are modeled as in [8], and the fitting parameters have been extracted by comparing the analytical model with a large set of experiments for bulk MOSFETs in the three considered crystallographic orientations (see Fig. 6). Finally, a mobility degradation with respect to the universal bulk-mobility curve is experimentally observed at low-medium $E_{\text {eff }}$ for (110)oriented SG-SOI FETs with $t_{\mathrm{Si}}=32 \mathrm{~nm}$ even if the device is essentially undoped (see Fig. 12). Recently, a number of publications reported the amount of interface states in devices with different orientations (e.g., [23], [24]) and indicated that (110)-oriented devices have an interface state density about three times larger than (100)-oriented devices. Moreover, it is known that the buried-oxide interface can influence mobility in UTB SOI-FETs [25]. Thus, when considering SOI-FETs, it is appropriate to model an additional contribution, which accounts for the mobility reduction induced by interface states, i.e.,

$$
\frac{1}{\tau_{\mathrm{it}, v}}=C_{\mathrm{it}_{0}}\left(\frac{N_{\mathrm{it}}}{N_{\mathrm{it0}}}\right)\left(\frac{N_{\text {inv0 }}}{N_{\mathrm{inv}}}\right)^{\zeta} .
$$

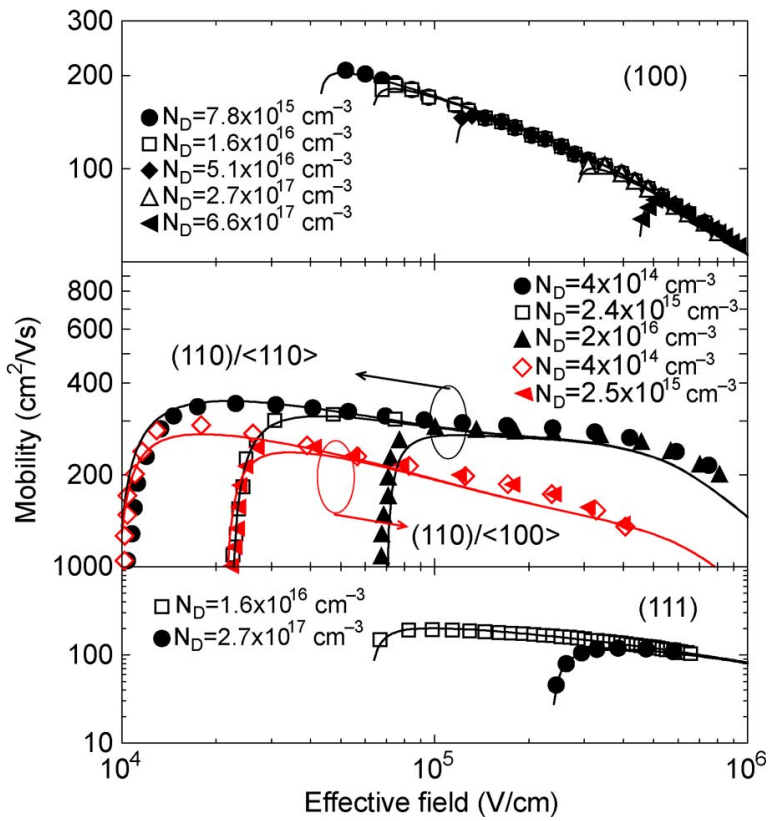

Fig. 6. Hole mobility in (100), (110), and (111) bulk MOSFETs versus effective field for various substrate doping concentrations. (Symbols) Experiments of (100) from [19], (110) from [20], and (111) from [21] and [22]. (Solid lines) Our model.

where $N_{\mathrm{it} 0}=5 \times 10^{10} \mathrm{~cm}^{-2}, \zeta=0.5$ as reported in the literature, and $C_{\mathrm{it}}$ is a constant extracted from the comparison with experiments. A value of $1.5 \times 10^{11} \mathrm{~cm}^{-2}$ (similar to that experimentally shown in [26]) has been used to reproduce the experiments by Tsutsui et al. [7]. Considering that, in a (100)oriented device, $N_{\mathrm{it}}$ is typically of the order of $5 \times 10^{10} \mathrm{~cm}^{-2}$ [26], the above scattering contribution is found to be effective only in the (110) case. $N_{\text {it }}$ should be considered an effective interface state density also accounting for the back interface defects.

Different from electrons [9], the hole mobility curves at low temperature reported in [7] are independent of $t_{\mathrm{Si}}$ down to $9 \mathrm{~nm}$. Thus, no dependence on $t_{\mathrm{Si}}$ has been modeled in (10). For $t_{\mathrm{Si}}<9 \mathrm{~nm}$, additional physical effects become relevant and contribute to degrade mobility, as explained in the following.

\section{BAND STRUCTURES AND REPOPUlation EFFECTS}

In order to calculate the relative populations of the $\mathrm{LH}$ and $\mathrm{HH}$ valleys in samples with different $t_{\mathrm{Si}}$ values and different orientations, we developed an analytical function based on physical considerations. Due to the lack of experimental data on (111) substrates, the mobility analysis is limited to the (100) and (110) ones. For zero normal electric field (quantum well), the analytical solution of the Schrödinger equation provides the expression for the energy levels. The relative distance between the $\mathrm{LH}$ and $\mathrm{HH}$ valley edges reads

$$
\Delta E_{\mathrm{VT}}=E_{\mathrm{VT}}^{\prime}-E_{\mathrm{VT}}=\frac{(\hbar \pi)^{2}}{2 t_{\mathrm{Si}}^{2}}\left(\frac{1}{m_{z, 1}}-\frac{1}{m_{z, 2}}\right) .
$$

The separation between the energy minima of the two valleys increases with the reduction of $t_{\mathrm{Si}}$, and holes mostly populate the $\mathrm{HH}$ valley. 


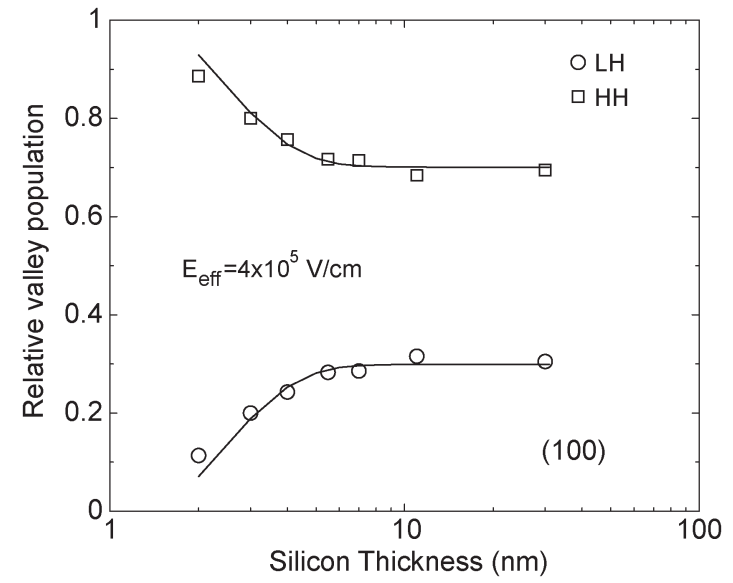

Fig. 7. Relative occupancy of the HH and LH valleys in (100)-oriented samples versus the silicon thickness at $E_{\text {eff }}=4 \times 10^{5} \mathrm{~V} / \mathrm{cm}$ from (2). (Symbols) Numerically calculated relative populations in [10]. (Lines) This model.

At large normal electric fields, the energy edges can be theoretically calculated, assuming a triangular potential well as in (1), and their difference is indicated with $\Delta E_{\mathrm{VE}}$ below. Finally, adopting the same formulation used for the conductionband edges in [8], the difference between the valley edges at high normal fields $\Delta E_{V}$ can be expressed as

$$
\Delta E_{V}=\Delta E_{\mathrm{VT}}\left[1+\left(\Delta E_{\mathrm{VE}} / \Delta E_{\mathrm{VT}}\right)^{\beta}\right]^{1 / \beta}
$$

where $\beta=3.5$. In order to validate (12), the relative-valley populations for (100) FETs have been calculated by using (2) as a function of $t_{\mathrm{Si}}$ and compared with those reported in [10] (see Fig. 7). A clear repopulation effect is evident at about $t_{\mathrm{Si}}=$ $7 \mathrm{~nm}$. The HH valley, i.e., the unprimed one, which exhibits the higher transport effective mass (see Table I), turns out to be repopulated for $t_{\mathrm{Si}}<7 \mathrm{~nm}$, with a negative effect on mobility. This effect contributes to the monotonic mobility degradation with decreasing $t_{\mathrm{Si}}$, as shown in Section V. As far as the (110) orientation is concerned, the same formulation in (12) has been used. However, in this case, the repopulation effect does not remarkably influence the hole mobility since the $\mathrm{HH}$ valley is almost entirely populated even at very thick silicon films and bulk MOSFETs (see Fig. 3).

\section{Model Enhancements for $t_{\mathrm{Si}}<5 \mathrm{~nm}$}

\section{A. (100) Substrates}

1) Scattering Induced by Silicon-Thickness Fluctuations: The measured (100) mobility for $t_{\mathrm{Si}}<5 \mathrm{~nm}$ exhibits a strong degradation that was ascribed to the scattering induced by $t_{\mathrm{Si}}$ fluctuations [27]. The formulation in [9] is used here to

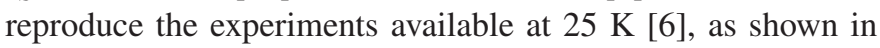
Fig. 8 (top).

2) Suppression of Intersubband Phonon Scattering: This effect should not take place in (100) FETs because the energy difference between the $\mathrm{HH}$ and $\mathrm{LH}$ valleys is lower than the energy of the $f$-type phonons even at high $E_{\text {eff }}$ (see Fig. 2), thus forbidding the suppression of the intersubband phonon scattering. This is confirmed by the experimental mobility in

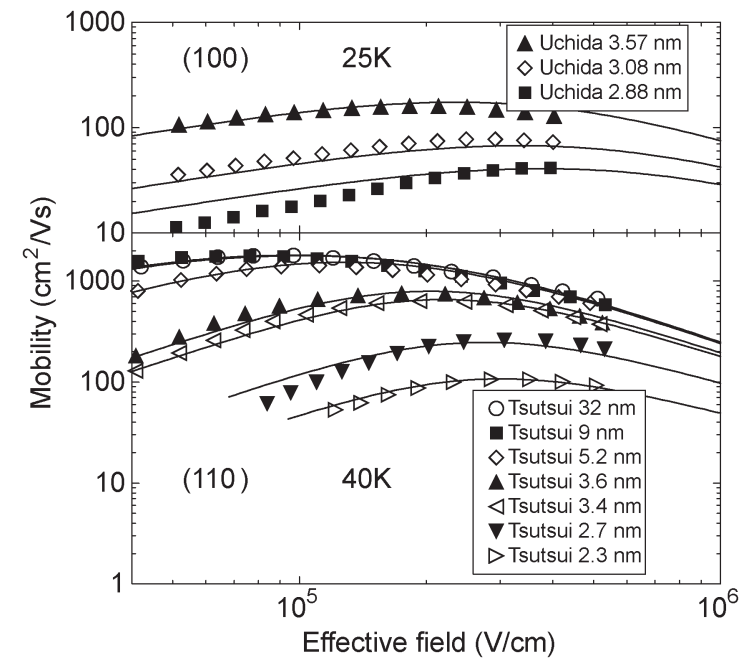

Fig. 8. Hole mobility versus effective field at low temperature for (top) (100)and (bottom) $(110) /\langle 110\rangle$-oriented FETs. (Symbols) Measurements from [6] and [7]. (Solid lines) Coulomb + surface-roughness + silicon thicknessfluctuation limited mobility. The phonon scattering contribution depends on the temperature as $T^{1.75}$ [10]; thus, its influence on mobility at low temperature is quite negligible (1\% and $3 \%$ phonon scattering at room temperature at 25 and $40 \mathrm{~K}$, respectively).
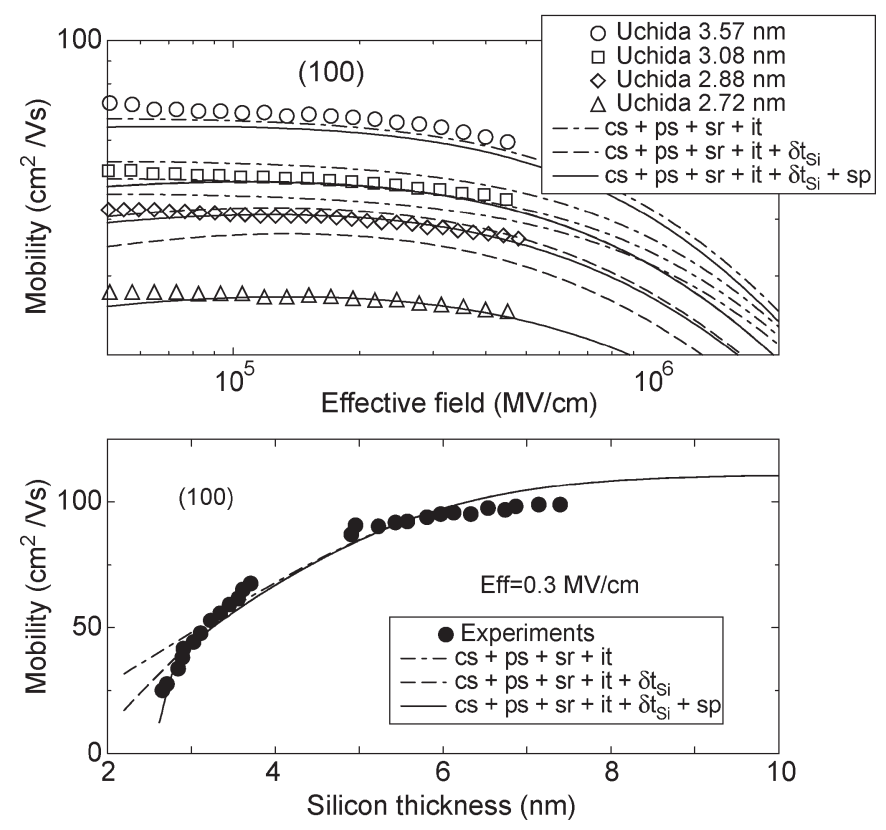

Fig. 9. Hole mobility versus (top) effective field and (bottom) silicon thickness at $300 \mathrm{~K}$ for (100) devices. (Symbols) Measurements from [6]. (Dashed lines) Coulomb + phonon + surface-roughness limited mobility. (Long dashed lines) Coulomb + phonon + surface-roughness + thickness-fluctuation limited mobility. (Solid lines) Coulomb + phonon + surface-roughness + thicknessfluctuation + surface optical-phonon-limited mobility.

(100) substrates reported in Fig. 9 (bottom), where the curve monotonically decreases by shrinking $t_{\mathrm{Si}}$.

3) Surface Optical Phonons: In order to reproduce the mobilities of devices thinner than $3 \mathrm{~nm}$ at $300 \mathrm{~K}$, we introduced the surface-mode optical-phonon scattering contribution [4]. The effects of the above scattering terms are highlighted in Fig. 9 (top). 

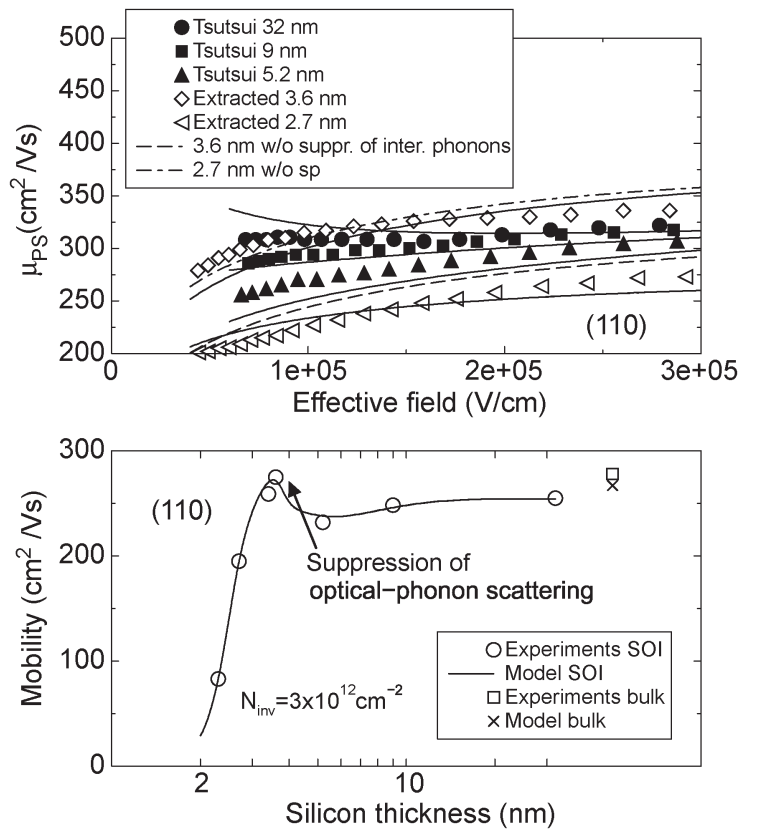

Fig. 10. Phonon-limited mobility in $(110) /\langle 110\rangle$ FETs versus (top) effective field and versus (bottom) silicon thickness for small silicon thicknesses. (Symbols) Measurements from [7]. The impact of the suppression on intervalley optical phonons and of the surface optical phonons are highlighted.

\section{B. (110) Substrates}

1) Scattering Induced by Silicon-Thickness Fluctuations: The same model has been used for the (110) case as well, with parameters extracted by the comparison with experiments carried out at $40 \mathrm{~K}$ by Tsutsui et al. [7] [see Fig. 8 (bottom)].

As explained in Section III, when the (110) p-FET is considered, the impact of optical-phonon scattering becomes nontrivial, and its dependence on $t_{\mathrm{Si}}$ should be correctly modeled. In [7], the phonon-scattering limited mobility has been extracted by means of Matthiessen's rule and by assuming that only the phonon contribution would remarkably change with temperature. However, this is incorrect because the scattering induced by thickness fluctuations varies with temperature as well. Indeed, the phonon-limited mobility extracted in [7] for $t_{\mathrm{Si}}<5 \mathrm{~nm}$ results to be negative for $N_{\mathrm{inv}}<10^{12} \mathrm{~cm}^{-2}$. Here, the temperature dependence of the MRTs relative to $t_{\mathrm{Si}}$ fluctuations has been assumed to be equal in the (100) and (110) FETs. Thus, in order to correctly model the phonon-limited mobility in the ultrathin (110) SOI, the latter has been reextracted from the experiments and used as a reference for the model validation [see open symbols in Fig. 10 (top)].

2) Suppression of the Intervalley Phonon Scattering: As anticipated in Section III, the absorbtion of optical phonons between the lowest $\mathrm{HH}$ and $\mathrm{LH}$ subbands plays a role at low fields, whereas it is suppressed at high $E_{\text {eff }}$ values. The simplest way to introduce the suppression of intervalley phonon scattering is to model a reduction of the inverse MRT (9) as a function of $\Delta E_{V}$, i.e.,

$$
\frac{1}{\tau_{\mathrm{OP}, 2}^{\prime}}=\frac{1}{\tau_{\mathrm{OP}, 2}}\left(1-f\left(\Delta E_{V}\right)\right) \text {. }
$$

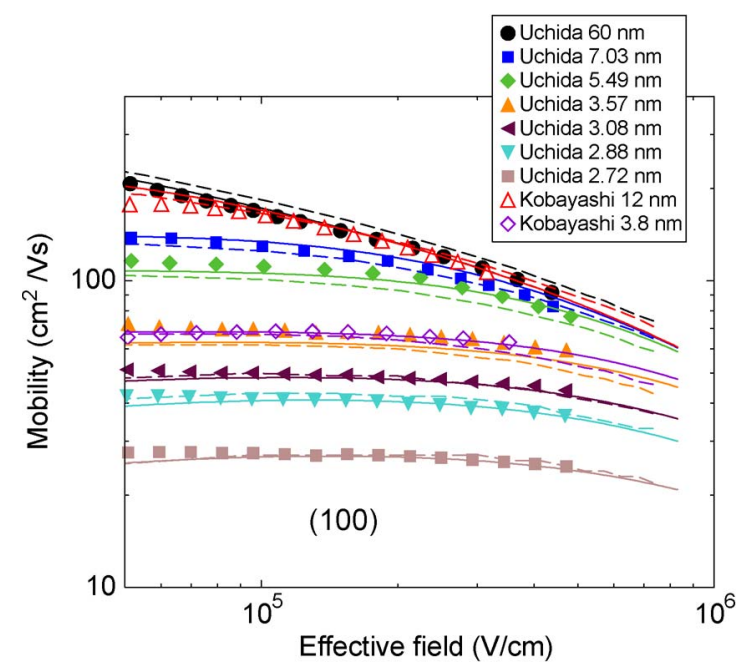

Fig. 11. Hole mobility in (100) FETs versus effective field for a wide range of silicon thicknesses at $300 \mathrm{~K}$. (Solid lines) Effective mobility model. (Dashed lines) Simulation results obtained using the local mobility model. (Symbols) Measurements from [6] and [28].

By exploiting the subband structure model presented in Section IV, $f\left(\Delta E_{V}\right)$ in (13) is modeled as

$$
f\left(\Delta E_{V}\right)=\frac{f_{0}}{1+\exp \left[-\left(\Delta E_{V}-\Delta E_{\mathrm{V} 0}\right) / C_{\mathrm{iv}}\right]}
$$

where $f_{0}, \Delta E_{V 0}$, and $C_{\text {iv }}$ are the fitting parameters calibrated on the experiments by [7] at $N_{\mathrm{inv}}=3 \times 10^{12} \mathrm{~cm}^{-2}$, as depicted in Fig. 10 (bottom). Thus, the mobility enhancement observed in [7] can be nicely reproduced by the above approach. $f\left(\Delta E_{V}\right)$ becomes significantly different from zero for $\Delta E_{V}>60 \mathrm{meV}$. Another important observation is that, according to (13) and (14), the mobility enhancement in the (110) samples is expected to increase as $E_{\text {eff }}$ increases because of the $\Delta E_{V}$ growth with $E_{\text {eff }}$ (see Fig. 2). However, in very thin-film FETs (below $5 \mathrm{~nm}$ ), additional scattering mechanisms limit the carrier mobility, i.e., the scattering induced by $t_{\mathrm{Si}}$ fluctuations (presented in Section IV) and the scattering with surface optical phonons (described below).

3) Surface Optical Phonons: The same expression for the conventional orientation is also used in this case. The model parameters are calibrated directly on the experiments at $300 \mathrm{~K}$. The effects of this scattering term is shown in Figs. 10 (top) and 12 .

\section{Review of the Complete Mobility Model}

All the scattering terms described above need to be accounted for in (7) to calculate the effective mobility. When bulk MOSFETs are considered, $1 / \tau_{\mathrm{it}, v}$ should be neglected. Figs. 11 and 12 compare the predictions of the complete mobility model with the complete set of measurement data. For implementation reasons, drift-diffusion device simulators require a mobility model dependent on the local carrier concentration $n$ and transverse electric field $E_{\perp}$, rather than the nonlocal inversionlayer carrier density and effective field. To this purpose, $N_{\text {inv }}$ and $E_{\text {eff }}$ are simply replaced by $n$ and $E_{\perp}$ in our model, and the fitting parameters are reextracted by the comparison with 


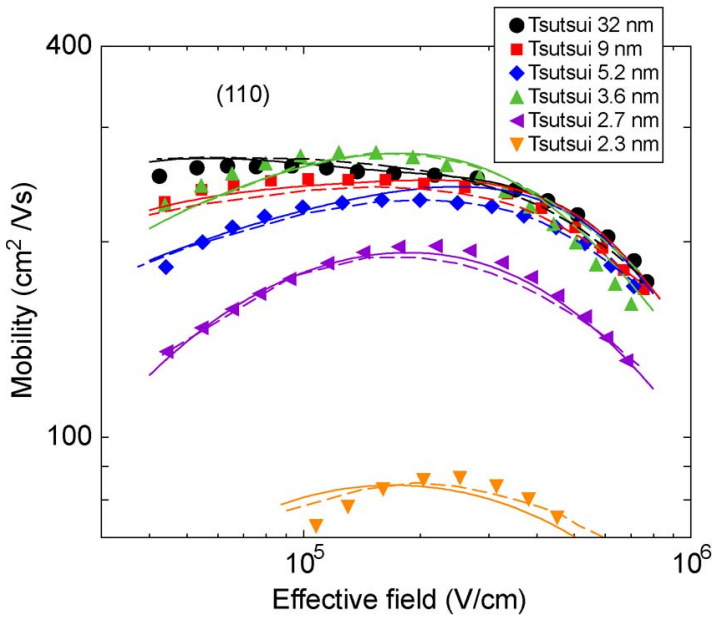

Fig. 12. Hole mobility in $(110) /\langle 110\rangle$ FETs versus effective field for a wide range of silicon thicknesses at $300 \mathrm{~K}$. (Solid lines) Effective mobility model. (Dashed lines) Simulation results obtained using the local mobility model. (Symbols) Measurements from [7].

the experiments. Figs. 11 and 12 show the comparison between the measurements and the local model (dashed lines) for the complete set of measurement data in [6], [7], and [28] for SGSOI FETs as a function of $E_{\text {eff }}$ and $t_{\mathrm{Si}}$. The effective mobility is also shown with solid lines. The performance of the local mobility model is good, the maximum relative error being $13 \%$. This partly confirms the qualitative results obtained in [29].

\section{CONCLUSION}

A low-field hole mobility model suitable for devicesimulation tools in (100) and (110) UTB SOI MOSFETs has been developed and calibrated on a wide set of experimental data. The model accounts for the main physical effects related to the quantum-mechanical structural confinement and transposes them into simple analytical formulations. The inclusion of silicon-thickness fluctuations and surface-phonon scattering extends the validity of the model to very small silicon thicknesses. Moreover, accounting for the scattering induced by interface states and the impact of the suppression of intervalley optical-phonon scattering, the model is able to reproduce (100) and (110) mobilities.

The experimental data on the (100) and (110) cases are reproduced in the model with a maximum error of about $10 \%-15 \%$.

\section{REFERENCES}

[1] International Technology Roadmap for Semiconductors 2007 Edition. [Online]. Available: http://public.itrs.net

[2] S. Mehrotra, A. Paul, M. Luisier, and G. Klimeck, "Surface and orientation dependence on performance of trigated silicon nanowire pMOSFETs," in Proc. IEEE Workshop Microelectron. Electron Devices, 2009, pp. 1-4.

[3] B. Yu, L. Chang, S. Ahmed, H. Wang, S. Bell, C. Yang, C. Tabery, C. Ho, Q. Xiang, T. King, J. Bokor, C. Hu, M. Lin, and D. Kyser, "FINFET scaling to $10 \mathrm{~nm}$ gate length," in IEDM Tech. Dig., 2002, pp. 251-254.

[4] S. Reggiani, E. Gnani, A. Gnudi, M. Rudan, and G. Baccarani, "Lowfield electron mobility model for ultrathin-body SOI and double-gate MOSFETs with extremely small silicon thicknesses," IEEE Trans. Electron Devices, vol. 54, no. 9, pp. 2204-2212, Sep. 2007.

[5] C. Kampen, A. Burenkov, J. Lorenz, H. Ryssel, V. Aubry-Fortuna, and A. Bournel, "An application-driven improvement of the drift-diffusion model for carrier transport in decanano-scaled CMOS devices," IEEE Trans. Electron Devices, vol. 55, no. 11, pp. 3227-3235, Nov. 2008.

[6] K. Uchida, H. Watanabe, A. Kinoshita, J. Koga, T. Numata, and S. Takagi, "Experimental study on carrier transport mechanisms in ultrathin-body SOI n- and p-MOSFETs with SOI thicknesses less than $5 \mathrm{~nm}$," in IEDM Tech. Dig., 2002, pp. 47-50.

[7] G. Tsutsui, M. Saitoh, and T. Hiramoto, "Experimental study on superior mobility in (110)-oriented UTB SOI pMOSFETs," IEEE Electron Device Lett., vol. 26, no. 11, pp. 836-838, Nov. 2005.

[8] L. Silvestri, S. Reggiani, E. Gnani, A. Gnudi, and G. Baccarani, "A low-field mobility model for bulk, ultra-thin body SOI and doublegate n-MOSFETs with different surface and channel orientations. Part I-Fundamental principles," IEEE Trans. Electron Devices, vol. 57, no. 7, pp. 1567-1574, Jul. 2010.

[9] L. Silvestri, S. Reggiani, E. Gnani, A. Gnudi, and G. Baccarani, "A low-field mobility model for bulk, ultra-thin body SOI and double-gate n-MOSFETs with different surface and channel orientations. Part II-Ultra-thin silicon films," IEEE Trans. Electron Devices, vol. 57, no. 7, pp. 1575-1582, Jul. 2010.

[10] M. V. Fischetti, Z. Ren, P. M. Solomon, M. Yang, and K. Rim, "Sixband $\mathrm{k} \cdot \mathrm{p}$ calculation of the hole mobility in silicon inversion layers: Dependence on surface orientation, strain, and silicon thickness," J. Appl. Phys., vol. 94, no. 2, pp. 1079-1095, Jul. 2003.

[11] A. Pham, C. Jungemann, and B. Meinerzhagen, "Physics-based modeling of hole inversion-layer mobility in strained-SiGe-on-insulator," IEEE Trans. Electron Devices, vol. 54, no. 9, pp. 2174-2182, Sep. 2007.

[12] L. Donetti, F. Gamiz, and N. Rodriguez, "Simulation of hole mobility in two-dimensional systems," Semicond. Sci. Technol., vol. 24, no. 3, p. 035016, Feb. 2009.

[13] E. Gnani, A. Gnudi, S. Reggiani, and G. Baccarani, "Effective mobility in nanowire FETs under quasi-ballistic conditions," IEEE Trans. Electron Devices, vol. 57, no. 1, pp. 336-344, Jan. 2010.

[14] F. Stern and E. Howard, "Properties of semiconductor surface inversion layers in the electric quantum limit," Phys. Rev., vol. 163, no. 3, pp. 816835, Nov. 1967.

[15] M. D. Michielis, D. Esseni, Y. L. Tsang, P. Palestri, L. Selmi, A. G. O'Neill, and S. Chattopadhyay, "A semianalytical description of the hole band structure in inversion layers for the physically based modeling of pMOS transistors," IEEE Trans. Electron Devices, vol. 54, no. 9, pp. 2164-2173, Sep. 2007.

[16] A. Nathan and H. Baltes, Microtrasducer CAD—Physical and Computational Aspects. New York: Springer-Verlag, 1999, ser. Computational Microelectronics.

[17] S. Dhar, H. Kosina, V. Palankovsky, E. Ungersboeck, and S. Selberherr, "Electron mobility model for strained-Si devices," IEEE Trans. Electron Devices, vol. 52, no. 4, pp. 527-533, Apr. 2005.

[18] E. Gnani, S. Reggiani, M. Rudan, and G. Baccarani, "On the electrostatics of double-gate and cylindrical nanowire MOSFETs," J. Comput. Electron., vol. 4, no. 1/2, pp. 71-74, 2005.

[19] S. Takagi, A. Turiumi, M. Iwase, and H. Tango, "On the universality of inversion layer mobility in Si MOSFETs: Part II-Effects of surface orientation," IEEE Trans. Electron Devices, vol. 41, no. 12, pp. $2363-$ 2368, Dec. 1994

[20] M. Saitoh, S. Kobayashi, and K. Uchida, "Physical understanding of fundamental properties of $\mathrm{Si}$ (110) pMOSFETs inversion-layer capacitance, mobility universality, and uniaxial stress effects," in IEDM Tech. Dig., 2007, pp. 711-714.

[21] H. Irie, K. Kita, K. Kyuno, and A. Toriumi, "In-plane mobility anisotropy and universality under uni-axial strains in n- and p-MOS inversion layers on (100), (110), and (111) Si," in IEDM Tech. Dig., 2004, pp. 9.5.1-9.5.4.

[22] B. Mereu, C. Rossel, E. P. Gusev, and M. Yang, "The role of Si orientation and temperature on the carrier mobility in metal oxide semiconductor field-effect transistors with ultrathin $\mathrm{HfO}_{2}$ gate dielectrics," J. Appl. Phys., vol. 100, no. 1, pp. 014504-1-014504-6, Jul. 2006.

[23] M. Yang, V. W. C. Chan, K. K. Chan, L. Shi, D. M. Fried, J. H. Stathis, A. I. Chou, E. Gusev, J. A. Ott, L. E. Burns, M. V. Fischetti, and M. Ieong, "Hybrid-orientation technology (HOT): Opportunities and challenges," IEEE Trans. Electron Devices, vol. 53, no. 5, pp. 965-978, May 2006.

[24] L. Trojman, L. Pantisano, I. Ferain, S. Severi, H. Maes, and G. Goeseneken, "Mobility and dielectric quality of 1-nm EOT HfSiON on Si(110) and (100)," IEEE Trans. Electron Devices, vol. 55, no. 12, pp. 3414-3420, Dec. 2008.

[25] J. Koga, S. Takagi, and A. Toriumi, "Influences of buried-oxide interface on inversion-layer mobility in ultra-thin SOI MOSFETs," IEEE Trans. Electron Devices, vol. 49, no. 6, pp. 1042-1048, Jun. 2002.

[26] B. Goebel, D. Schumann, and E. Bertagnolli, "Vertical N-channel MOSFETs for extremely high density memories: The impact of 
interface orientation on device performance," IEEE Trans. Electron Devices, vol. 48, no. 5, pp. 897-906, May 2001.

[27] D. Esseni, A. Abramo, L. Selmi, and E. Sangiorgi, "Physically based modeling of low field electron mobility in ultrathin single- and doublegate SOI n-MOSFETs," IEEE Trans. Electron Devices, vol. 50, no. 12, pp. 2445-2455, Dec. 2003.

[28] S. Kobayashi, M. Saitoh, and K. Uchida, "More-than-universal mobility in double-gate SOI p-FETs with sub-10-nm body thickness-role of lighthole band and compatibility with uniaxial stress engineering," in IEDM Tech. Dig., 2007, pp. 707-710.

[29] A. Schenk, "A local mobility model for ultra-thin DGSOI nMOSFETs," in Proc. SISPAD, 2004, pp. 113-116.

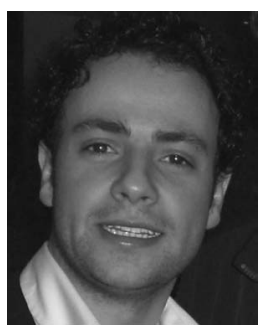

Luca Silvestri received the B.S. and M.S. degrees in electrical engineering from the University of Bologna, Bologna, Italy, in 2004 and 2007, respectively.

Since May 2007, he has been with the Ercole De Castro Advanced Research Center for Electronic Systems (ARCES), University of Bologna, and since January 2008, he has been with the European Doctorate program in information technology at ARCES, working on the development of physical models and simulation tools for nanoscale devices. Since October 2009, he has been a Visiting Researcher with the Microwave Laboratory, Université Catholique de Louvain, Louvain-la-Neuve, Belgium, working on the modeling and the simulation of silicon nanowires for sensor applications.

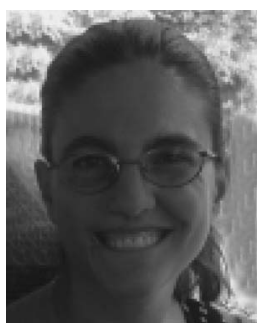

Susanna Reggiani (M'07) received the Laurea and $\mathrm{Ph} . \mathrm{D}$. degrees in electrical engineering from the University of Bologna, Bologna, Italy, in 1997 and 2001, respectively.

Since April 1997, she has been with the Department of Electronics, Computer Science and Systems (DEIS), University of Bologna, working on the numerical simulation of semiconductor devices and the simulation of electron transport in nanoscale devices applied to quantum computing. In 2001, she was a Research Associate with the University of Bologna, where she is currently with the Ercole De Castro Advanced Research Center for Electronic Systems.

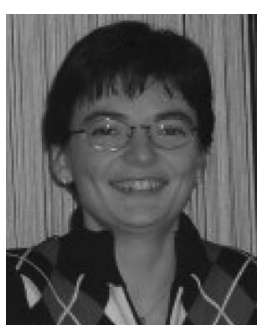

Elena Gnani (M'98) received the Laurea and Ph.D. degrees in electrical engineering from the University of Bologna, Bologna, Italy, in 1999 and 2003, respectively.

Since October 1999, she has been with the Department of Electronics, Computer Science and Systems (DEIS), University of Bologna, working on the physics of carrier transport and the numerical analysis of semiconductor devices. She is also currently with the Ercole De Castro Advanced Research Center for Electronic Systems, University of Bologna.

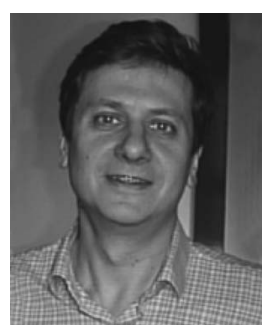

Antonio Gnudi (M'98) received the Laurea degree in electrical engineering and the Ph.D. degree in computer science from the University of Bologna, Bologna, Italy, in 1983 and 1989, respectively.

From 1989 to 1990 , he was a Visiting Scientist with the IBM T.J. Watson Research Center, Yorktown Heights, NY, engaged in the development of advanced physical models and numerical simulation tools for charge transport in semiconductor devices. In 1990, he became a Research Assistant and, in 1998, an Associate Professor of electronics with the University of Bologna, where he was involved in research and teaching activities concerning the design of analog complimentary metal-oxide-semiconductor circuits for RF applications and the modeling, the design, and the characterization of RF microelectromechanical-system devices for wireless applications. His current research interests include the numerical simulation of nanometric devices, including semiclassical and full-quantum transport, and efficient algorithms for their solutions.

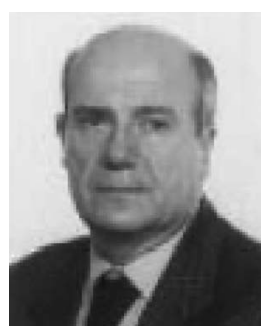

Giorgio Baccarani (S'68-M'80-SM'92-F'00) received the Dr.Ing. degree in electrical engineering and the Ph.D. degree in physics from the University of Bologna, Bologna, Italy, in 1967 and 1969, respectively.

In 1969, he joined the Bell Laboratories, Murray Hill, NJ, as a limited-term Member of the Technical Staff, working in the area of electron-device processing. In 1970, he became a Research Assistant with the University of Bologna, where he investigated the physical properties of MOS structures and transport effects in semiconductor materials and devices. Since 1972, he has been teaching an annual course on quantum electronics. In 1980, he became a Full Professor of digital electronics with the University of Bologna. In 1981, he was on a one-year assignment with the IBM Thomas J. Watson Research Center, Yorktown Heights, NY, where he investigated the feasibility of a one-fourth micrometer MOS process from the standpoint of the physical limitations affecting the device performance. In 1983 and 1989, he was again a Visiting Scientist with IBM, investigating advanced physical models for device simulation and silicon-based heterojunction bipolar transistors, respectively. Since 1983, he has been heading a group involved in the numerical analysis of semiconductor devices, acting as a Partner Leader in the context of several projects supported by the European Economic Community in the area of computer-aided design for very large scale integration. $\mathrm{He}$ is one of the initial proposers and the Director of the Ercole De Castro Advanced Research Center for Electronic Systems, University of Bologna, founded in 2001. His current research interests include numerical-device simulation, synthesis of analog circuits, analog and digital architectures for image processing, and integrated-circuit design. 